\title{
Terahertz Imaging via Electrooptic Effect
}

\author{
Zhiping Jiang and Xi-Cheng Zhang, Senior Member, IEEE
}

\begin{abstract}
In this paper, we describe and discuss three electrooptic-based terahertz imaging systems: the point scanning system, a two-dimensional charge-coupled device (CCD) system and a one-dimensional spatio-temporal chirped pulse imaging system. A complete comparison between the scanning system and CCD systems is given. We emphasize on the CCD systems that provide high acquisition speed by taking the unique advantages of parallel measurement. Possible improvements are also discussed.
\end{abstract}

Index Terms-CCD, chirped pulse measurement, electrooptic sampling, terahertz imaging, terahertz technique.

\section{INTRODUCTION}

$\mathbf{T}$ HE accessibility of electromagnetic waves in the terahertz range has opened an avenue for scientific research as well as practical applications. Historically, terahertz pulses were first generated and detected by photoconductive (PC) antennas. Using PC antennas as both the emitter and detector, terahertz imaging was realized by scanning the object [1], [2]. The imaging speed is obviously limited by the scanning speed, depending on the number of the pixels and integral time it may take (i.e., minutes or hours) to get one imaging picture, thus, it is difficult to realize real-time imaging. However, free-space electrooptic (EO) sampling [3]-[11] provides an alternative method. It has the advantages of easy alignment, wide bandwidth, high sensitivity, and most importantly, the capability of parallel measurement. In this paper, we will describe and compare three terahertz imaging systems based on EO effects and study the possible improvements for the charge-coupled device (CCD) systems. Throughout this paper, the laser used is the Coherent Ti:sapphire amplifier RegA 9000 , which produces $1-\mathrm{W}$ power, 250-fs pulses, 830-nm wavelength, and repetition rate of $250 \mathrm{kHz}$.

This paper is arranged as follows. In Sections II-IV, three imaging systems (the scanning system, two-dimensional (2-D) CCD system and chirped pulse measurement system) are described and typical images obtained by these systems are given. We introduce new detection geometry: crossed and balanced detection for EO sampling. To have a better understanding of the scanning system and the CCD imaging systems, a complete comparison is given in Section V. In Section VI, we describe some techniques that can improve the

Manuscript received March 26, 1999; revised July 7, 1999. This work was supported by the Army Research Office and by the Department of Energy under Contract DE-FG07-98ER62706.

Z. Jiang is with the ZOmega Technology Corporation, Latham, NY 12110 USA, and is also with the Rensselaer Polytechnic Institute, Troy, NY 12180 USA.

X.-C. Zhang is with the Physics Department, Rensselaer Polytechnic Institute, Troy, NY 12180 USA.

Publisher Item Identifier S 0018-9480(99)08468-9.

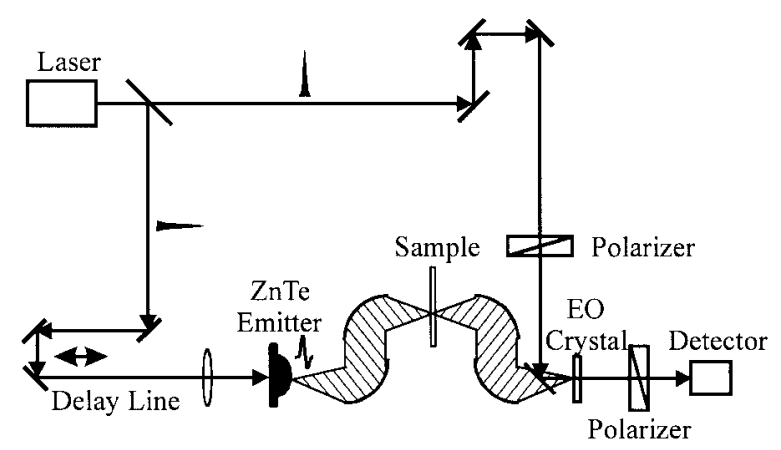

Fig. 1. Schematic of the scanning terahertz imaging system.

performances of the CCD system. We emphasize the emerging lock-in CCD, which could suppress the noise dramatically in the CCD system, and increase the signal-to-noise ratio to be comparable with the scanning system. Section VII presents a conclusion.

\section{SCANNIng Terahertz IMAGing SyStem}

The scanning terahertz imaging system described here is similar to that described in [1], except that both the emitter and receiver are based on the optical rectification and $\mathrm{EO}$ effect, as shown in Fig. 1. The emitter is a 2-mm $\langle 110\rangle \mathrm{ZnTe}$ crystal with a silicon ball attached to it to collimate the radiation; the receiver is a $4-\mathrm{mm}\langle 110\rangle \mathrm{ZnTe}$ crystal. Four parabolic mirrors with the numerical aperture of 0.5 are used to focus the terahertz beam. The dynamic range of this system can be as high as $5 \times 10^{4}$, the central frequency is around $0.8-0.9 \mathrm{THz}$. The scanning area can be $20 \times 20 \mathrm{~cm}^{2}$ with $1-\mu \mathrm{m}$ resolution. We also placed a galvanometer controlled time-delay stage in the pump beam, which allows us to do fast temporal scanning. A more detailed description can be found in [12]. Fig. 2(a)-(d) shows, respectively: (a) the typical temporal waveform and (b) its frequency distribution, the knife edge scanning of the focal spot gives: (c) 1.5-mm full width at half maximum (FWHM), and (d) the peak stability is about $0.3 \%$.

In this system, we normally use the modified detection geometry for EO sampling instead of the standard balance detection. Besides the balance detection, EO sampling can also be done with the two crossed polarizers. For the balance detection, it works at the linear point of the transmission curve, the common background currents and noise are cancelled by two identical detectors. It has the best linearity, but the smallest modulation depth. For the crossed polarizer detection, the dc laser background is blocked by the second crossed polarizer (analyzer) and, thus, this method has the biggest modulation depth. It is possible to take the advantages of both detections by using a "crossed plus balanced" detection, as shown in 


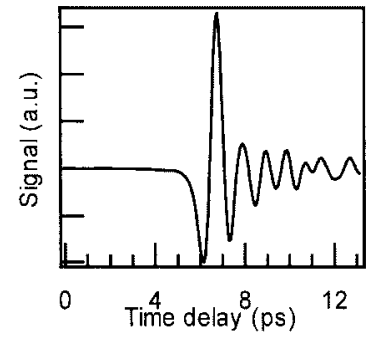

(a)

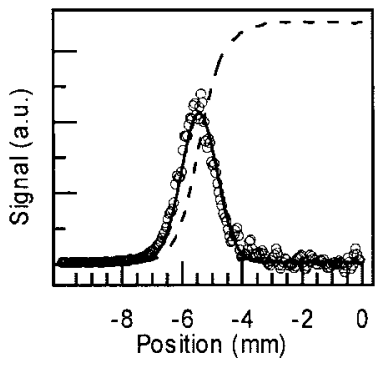

(c)

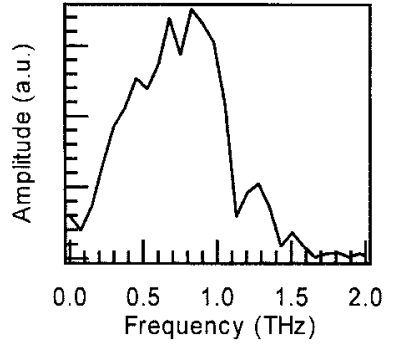

(b)

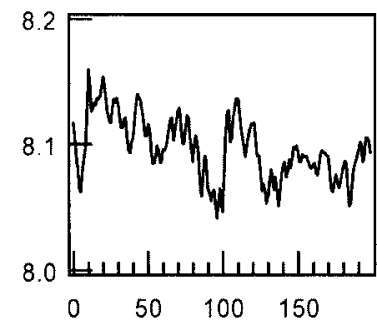

(d)
Fig. 2. (a) Typical terahertz temporal waveform and (b) its frequency distribution. (c) Knife edge scanning of the focal spot (dashed) and its derivative (circle). Gaussian fitting gives $1.5-\mathrm{mm}$ FWHM. (d) Stability of the peak signal. The rms fluctuation is about $0.3 \%$.

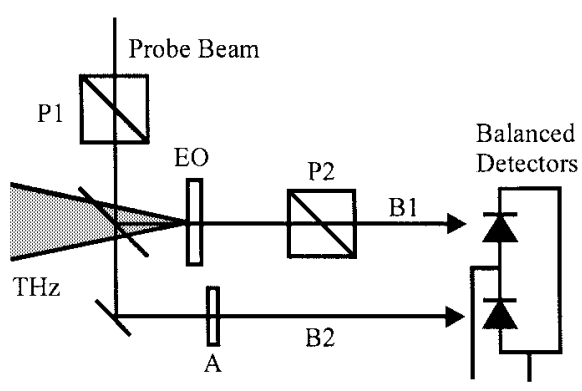

Fig. 3. "Crossed and balanced" detection for EO sampling. The transmission axis of the polarizer $P 2$ is perpendicular to that of $P 1$. The beam $B 1$ alone is the standard cross detection. Beam $B 2$, which has no terahertz signal, is used to cancel the laser fluctuation noise in $B 1$. A variable attenuator $A$ is used to adjust the $B 2$ intensity.

Fig. 3. The polarization of polarizer $P 2$ is perpendicular to that of $P 1$, thus, beam $B 1$ is the standard cross geometry. Obviously, we can use another beam $B 2$ to balance the common laser fluctuation. Here, we use attenuator $A$ to adjust the intensity of $B 2$ to be equal to that of $B 1$. As $B 2$ has no terahertz signal, it helps to reduce the laser fluctuation noise without changing the signal at all. If we get the beam $B 2$ after the EO crystal, and with the help of a compensator, we can have a negative signal in $B 2$, therefore, the signal can be increased by a factor of two.

To illustrate the possible applications of this system, we show a terahertz image of a mammographic phantom in Fig. 4. This phantom is used to evaluate the $\mathrm{X}$-ray imaging system. It contains five nylon fibers, five masses, and five groups of specks. All these structures are invisible to the naked eye. A good X-ray imaging system can see the third speck group, the fourth fiber, and the third mass. The scanning of the terahertz

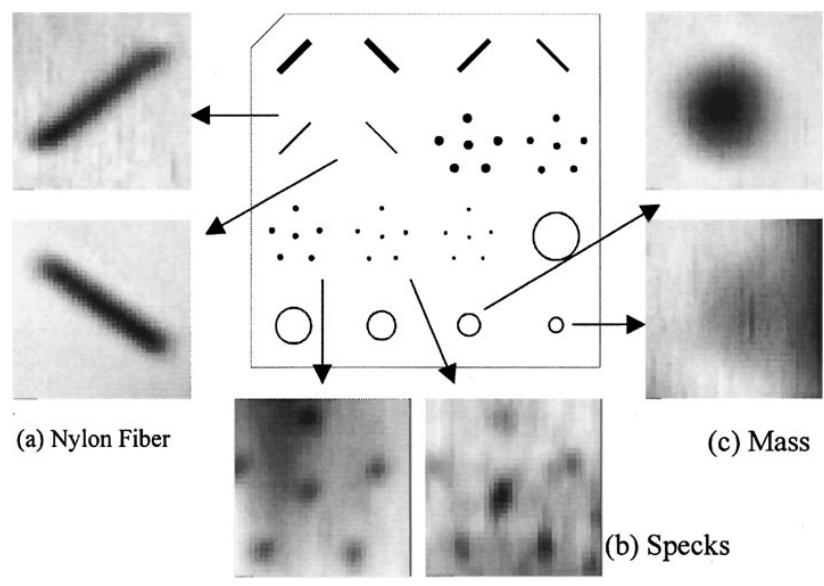

Fig. 4. Mammographic phantom and its terahertz images. The central diagram shows the structure, and the surrounding pictures are obtained with the terahertz imaging setup of Fig. 1.

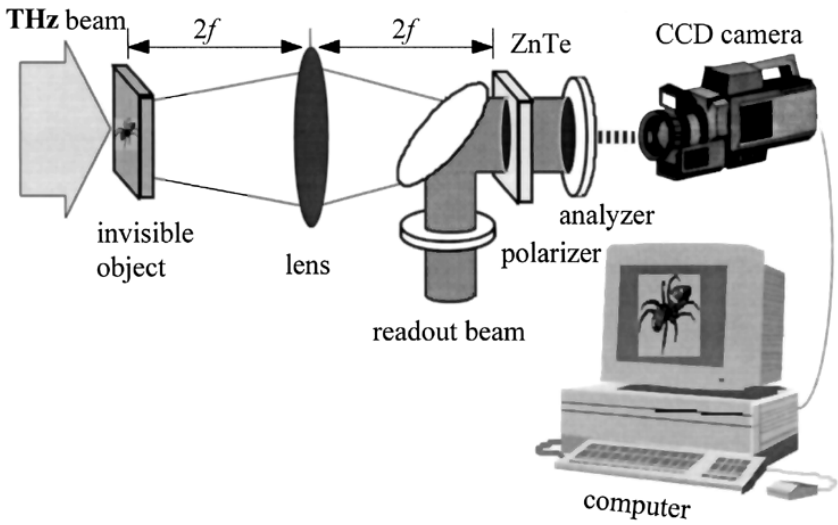

Fig. 5. 2-D real-time terahertz imaging system via EO effect.

imaging system can resolve the fifth fiber, the fifth mass, and the fourth speck group, indicating very good spatial resolution.

\section{2-D CCD IMAGING SYSTEM}

Fig. 5 shows the detection portion of a free-space EO terahertz imaging system with a CCD that has the capability of real-time imaging. The terahertz beam is generated by a large aperture photo-conductive switch triggered by the pump beam. A polyethylene lens is used to form the image of the object onto the $\langle 110\rangle$-oriented ZnTe crystal. An optical readout (probe) beam with a diameter larger than that of the terahertz image probes the electric-field distribution within the crystal via the Pockels effect. The 2-D terahertz field distribution in the sensor crystal is converted into a 2-D optical intensity modulation after the readout beam passes through a crossed polarizer (analyzer), the optical image is then recorded by a digital CCD camera. Unlike the conventional EO sampling, where the linear optical bias is used, two polarizers with nearly crossed polarizations are used (nearly zero optical bias) to increase the modulation depth.

Fig. 6 shows an example of the terahertz images taken by this system. This system is capable of noninvasive imaging of moving objects, turbulent flows, or hidden objects [13]-[15]. 


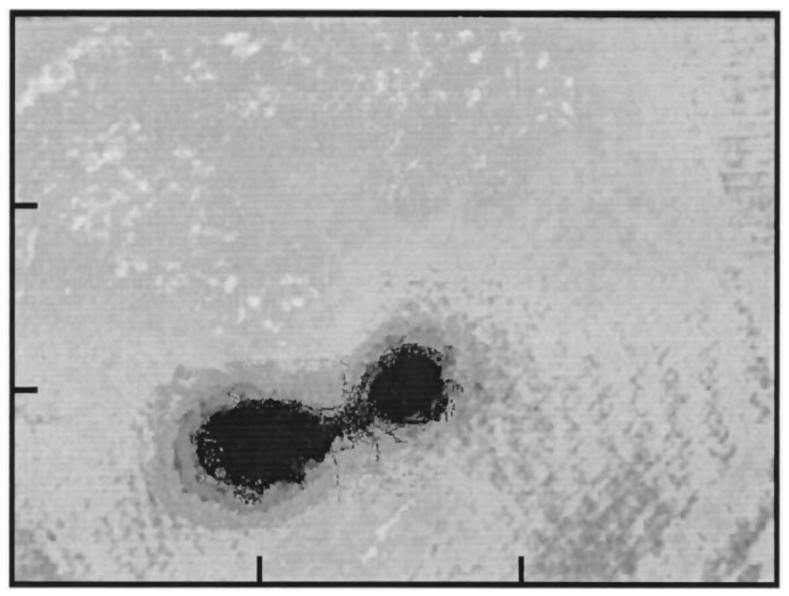

Fig. 6. Terahertz imaging of an ant taken by the CCD terahertz imaging system of Fig. 5.

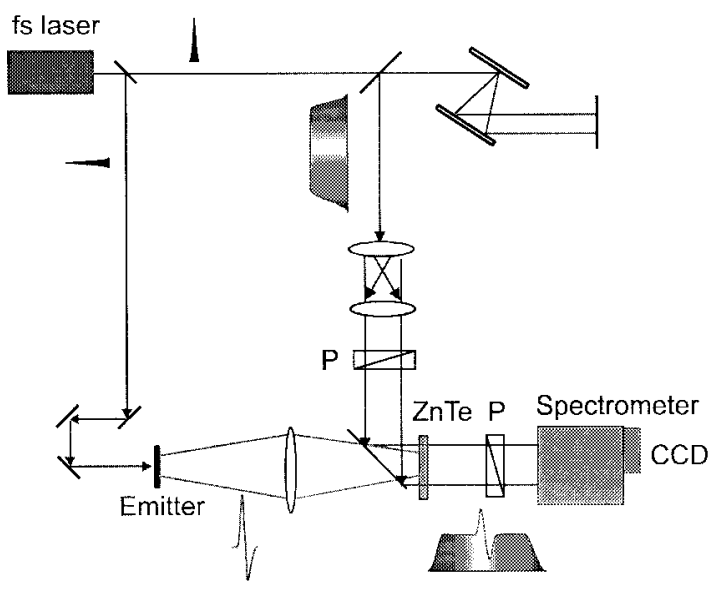

Fig. 7. Spatio-temporal terahertz imaging by a chirped probe pulse.

\section{ONE-DimENSIONAL SPATIO-TEMPORAL IMAGING}

Although the setup in Fig. 5 has real-time imaging capability, it can only obtain one image per time delay. An interesting alternative method is to use the chirped pulse technique, and the realization of spatial-temporal terahertz imaging is possible even without the mechanic time delay [16], [17]. Fig. 7 shows the schematic illustration of the chirped pulse technology, where the frequency of the probe beam is chirped and the pulse duration is stretched by a grating pair. When the chirped probe beam and a terahertz pulse co-propagate in the EO crystal, the different wavelength components of the chirped pulse are modulated by different portions of the terahertz pulse through the Pockels effect. Therefore, the terahertz waveform is encoded onto the wavelength spectrum of the probe beam. A spectrometer and a detector array (CCD) are used to measure the spectral distribution. We measured the spectral distributions with and without terahertz modulation, and the difference is proportional to the terahertz electrical field. Fig. 8 is an example showing the spatio-temporal imaging of a quadrupole terahertz emitter, the characteristic bipolar structure for each dipole is clearly seen, and the two backto-back dipoles have different polarities. If the EO crystal is

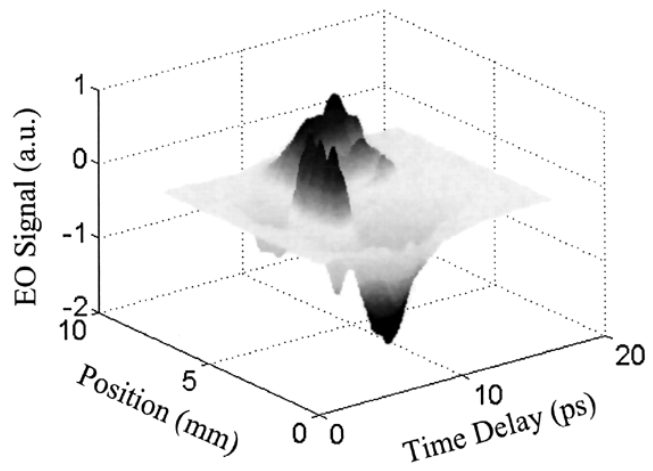

Fig. 8. Spatio-temporal terahertz imaging of a quadrupole.

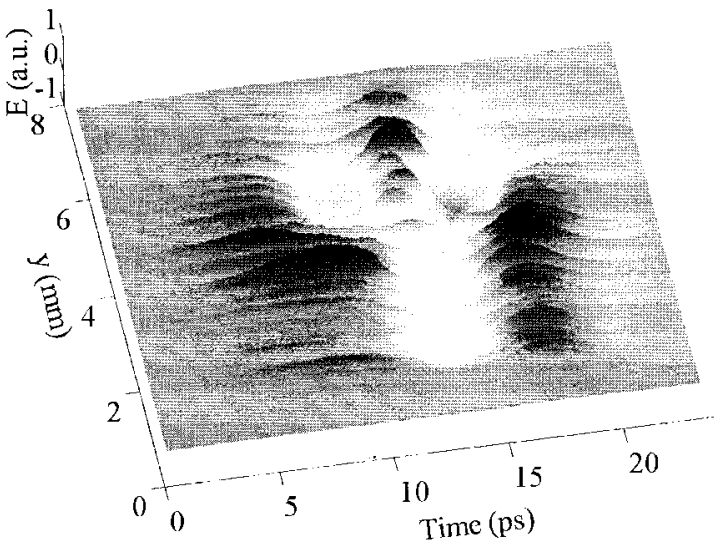

Fig. 9. Interference pattern between two back-to-back dipoles.

not right at the imaging plane, then we are able to observe the interference of these two dipoles, and the result is shown in Fig. 9. We can see the interference fringes and the polarity change along $y$-axis. In the experiment, the spatio-temporal distribution can be measured in real time. When we move the terahertz lens or the emitter, we are able to observe the spatiotemporal distribution dynamically on the computer screen. We have made some movies of the terahertz field while changing the imaging optics or moving the emitter. We should also note that no mechanic movement for the temporal delay and spatial scanning is needed. Actually, we are even able to obtain the image in a single-shot since every probe pulse contains the information of the whole terahertz pulse.

The chirped pulse technique is the only possible way for single shot spatio-temporal imaging of the terahertz electric field. It will find applications in some extreme situations where the conventional techniques are not able to apply. Possible applications include the study of the emitter breakdown, the measurement of the unsynchronized microwave and other unsynchronized phenomena, and nonlinear optical phenomena [18].

\section{Discussion}

To get a better understanding of the CCD terahertz imaging system, the discussion and comparison with the scanning system are necessary concerning the speed, detection geometry, signal-to-noise ratio (SNR), sensitivity, and uniformity. 


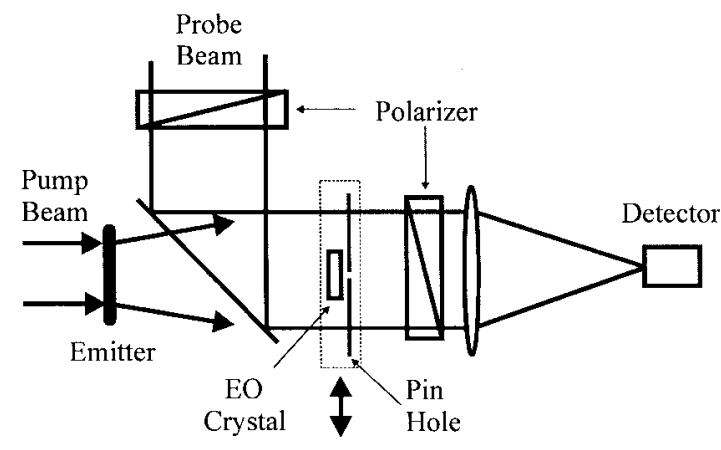

Fig. 10. Measurement of the terahertz spatio-temporal distribution by scanning the EO sensor. Since the expanded collimated probe beam is used, the relative timing of the terahertz and probe pulse is fixed.

1) Speed: For the point scanning system, the object under imaging is scanned mechanically and the speed is ultimately limited by this mechanic scanning. The upper limit may be on the order of 100 pixels per second, no matter how strong the terahertz source is. For the CCD terahertz imaging system, the upper limit is the CCD frame rate in continuous wave $(\mathrm{CW})$ detection mode, and laser pulse duration in single-shot mode. Therefore, the CCD imaging system is the only applicable system if the events are faster than tens of milliseconds.

2) Detection Geometry: In the scanning system, terahertz is focused down onto the object to a point as small as possible by nonimaging parabolic mirrors, and a single detector is used while scanning the object. In the CCD system, a collimated terahertz beam is illuminated onto the object, and an imaging lens is used to form the image of the object onto the EO crystal. A large probe beam with plane wavefront is used to readout the field distribution inside the EO crystal. If the object is the emitter itself, this geometry is ideal to measure the spatio-temporal distribution of the emitted terahertz field. Several examples are given in [12]. This geometry with wide probe beam is well suited to measure the spatio-temporal distribution of a terahertz electrical field by using one detector and scanning a small EO crystal. In this experiment, the CCD is replaced by one photodiode, and the probe beam is focused onto the detector. A small aperture is attached to the EO crystal. By scanning the aperture and EO crystal perpendicular to the probe beam, the spatial distribution is obtained without changing the timing (Fig. 10). The sensitivity is much higher than that using CCD. Fig. 11 shows the spatiotemporal distribution of a large aperture emitter $5 \mathrm{~cm}$ from the emitter [see Fig. 11(a)] and at the $2 f-2 f$ imaging plane [see Fig. 11(b)]. Both the amplitude distribution and wavefront (phase) are obtained. We also measured the diffraction of a circular aperture. In Fig. 11(c), the on-axis terahertz waveform is measured while changing the diameter of the aperture. The aperture is $6.7 \mathrm{~cm}$ away from the emitter and the measuring plane is $1.2 \mathrm{~cm}$ from the aperture. The position of the negative peak after the main peak has the quadratic behavior to the diameter of the aperture, which can be explained by the boundary diffraction wave [19] in time domain. Fig. 11(d) shows the spatio-temporal distribution at the measuring plane when the aperture diameter is $8.5 \mathrm{~mm}$. The boundary wave
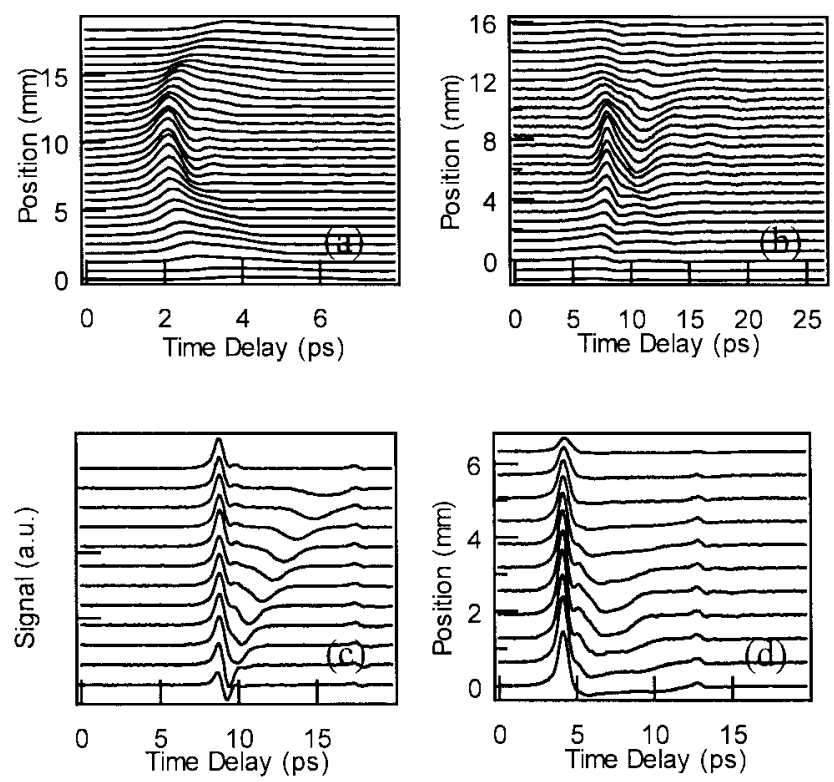

Fig. 11. Terahertz spatio-temporal distribution taken by scanning the EO crystal. The gap between two electrodes is about $8 \mathrm{~mm}$. (a) $5 \mathrm{~cm}$ from the emitter. (b) $2 f-2 f$ imaging plane with $f=5 \mathrm{~cm}$. (c) Diffraction of a circular aperture, from the bottom to the top curve (the aperture diameter varies from 2 to $12 \mathrm{~mm}$ with $1-\mathrm{mm}$ step). The top one is without aperture. (d) Spatial dependence (the aperture diameter is $8.5 \mathrm{~mm}$ ).

is well known, but hard to demonstrate with visible light; terahertz provides a easy way.

3) SNR: In the scanning system, a lock-in amplifier is used to depress the noise, therefore, the SNR is about three orders higher than that in the CCD system where the conventional lock-in amplifier cannot be used. The sensitivities of the scanning and CCD systems are about $1 \mathrm{mV} / \mathrm{m}$ and $1 \mathrm{~V} / \mathrm{m}$, respectively. Note that in the CCD system, the terahertz beam is large and collimated, thus, the electric field is smaller than in the scanning system. This makes the SNR even worse. However, the measurement is parallel in the CCD system, which allows a much longer integral time. Possible improvements are discussed in Section VI.

4) Uniformity: In the scanning system with a single detector, there is no uniformity problem for a 2-D imaging because the same detector is used for all the pixels. However, with a CCD system, there exist several sources that could influence the uniformity, including the profile of the laser probe beam, the quality of the CCD, and the homogeneity of the EO crystal. The first two can be easily corrected, but the EO crystal needs more consideration.

The origins of the inhomogeneity of the EO crystal are the residue birefringence and scattering. Let $I_{0}$ be the probe beam intensity, $\eta$ the scattering, $\Gamma_{0}$ the optical bias caused by the residue birefringence, and $\Gamma$ the phase proportional to the terahertz signal, then the transmitted light $I$ is given by the following relation [20]:

$$
I=I_{0}\left\lfloor\eta+\sin ^{2}\left(\Gamma_{0}+\Gamma\right)\right\rfloor \approx I_{0}\left\lfloor\eta+\Gamma_{0}^{2}+2 \Gamma_{0} \Gamma+\Gamma^{2}\right\rfloor .
$$

The background light is given by

$$
I_{b}=I_{0}\left(\eta+\Gamma_{0}^{2}\right)
$$



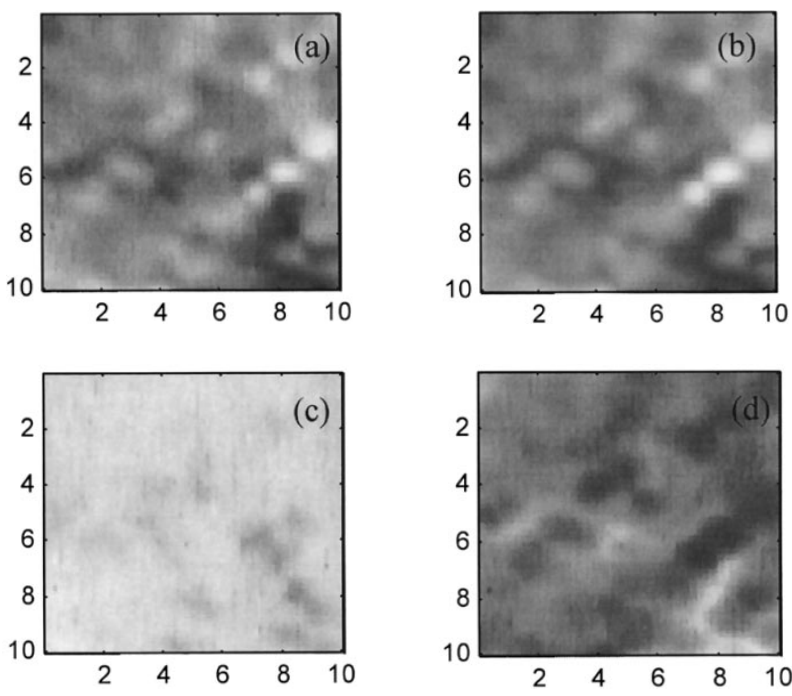

Fig. 12. Correction of the inhomogeneity of the EO crystal. (a) Terahertz signal $I_{s}$. (b) Background light $I_{b}$. (c) $I_{s} / \sqrt{I_{b}}$. (d) $I_{s} / I_{b}$. The uniformity of the corrected image [Fig. 12(c)] by (5) is much better than the uncorrected one [Fig. 12(a)].

and the terahertz signal is given by

$$
I_{s}=I_{0}\left(2 \Gamma_{0} \Gamma+\Gamma^{2}\right) .
$$

Since the scattering light $\eta$ and the residue birefringence $\Gamma_{0}$ are normally dependent on the spatial position, different EO crystal positions therefore give different background light and signal. This inhomogeneity deteriorates the image quality and needs to be corrected. It can be seen that there exists the following relation between the signal and background light:

$$
I_{s} \approx 2 \Gamma_{0} \Gamma I_{0} \approx 2 \sqrt{\frac{I_{b}}{I_{0}}} \Gamma I_{0}
$$

under the conditions of $\eta \ll \Gamma_{0}^{2}$ and $|\Gamma| \ll\left|\Gamma_{0}\right|$. In the right-hand-side term of (4), only the background light $I_{s}$ is position dependent. Therefore, the signal can be corrected by the following equation:

$$
I_{s}^{c}=\frac{I_{s}}{\sqrt{I_{b}}}
$$

where $I_{s}^{c}$ is the corrected signal. Equation (5) is used to correct the inhomogeneity of the EO crystal.

The uniformity of the EO crystal can be mapped out by scanning the EO crystal perpendicular to the probe beam. Fig. 12(a) and (b) shows the 2-D position dependence of the terahertz signal $I_{s}$ and the background light $I_{b}$, respectively. There is a strong similarity between these two pictures, because both inhomogeneities are due to $\Gamma_{0}$. Fig. 12(c) and (d) are $I_{s} / \sqrt{I_{b}}$ and $I_{s} / I_{b}$, respectively. Fig. 12(c) demonstrates much better uniformity than the original terahertz signal image [see Fig. 12(a)] and the ratio of Fig. 12(a) over Fig. 12(b). This shows that the inhomogeneity of the EO crystal mainly comes from the residue birefringence and that (5) is effective in compensating for this inhomogeneity.

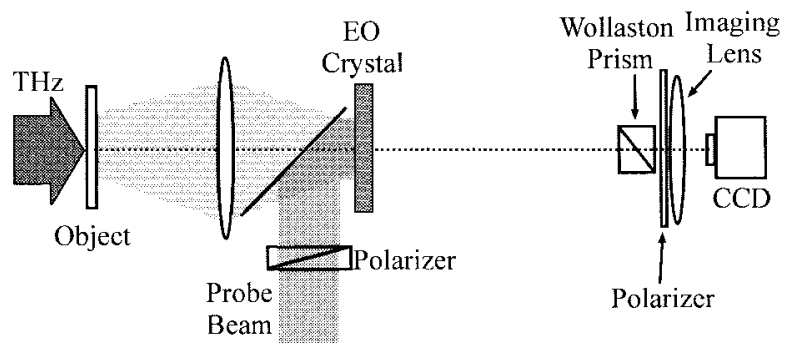

Fig. 13. Schematic of the CCD system with dynamic subtraction. A Wollaston prism splits the probe beam into two beams. These two beams are nearly identical, except for the intensities.

5) Resolution and Aberration: In principle, the spatial resolutions of both the scanning system and CCD system are determined by the wavelength and numerical aperture of the imaging system. Both systems would have similar spatial resolution if a similar terahertz source and numerical aperture of the imaging system are used. However, by taking the aberration and SNR into consideration, it turns out that the scanning system has a better spatial resolution. If the pointspread function is characterized (e.g., by using the setup in Fig. 10), it is then possible to enhance the spatial resolution by using deconvolution in the scanning system. In the CCD system, this is much harder to realize due to the inhomogeneity. Besides, since a large numerical aperture has to be used in order to increase the spatial resolution, the imaging may not obey the paraxial condition; it inevitably brings aberration into the image.

\section{IMPROVEMENTS}

As discussed in Section V, the SNR of the CCD system is lower than that of the scanning system; this is the main drawback of the terahertz imaging by using a CCD system. Below we will discuss several possible methods to improve the SNR in a CCD system.

1) Dynamic Subtraction: The major noise of the terahertz imaging comes from the laser intensity fluctuation, both spatially and temporally. In the conventional EO sampling, the spatial fluctuation does not contribute to the noise because the whole probe beam is focused onto the detectors. However, with a CCD camera, the spatial fluctuation does contribute to the noise. It can only be corrected if an identical reference picture of the same probe laser beam is taken. We have studied the capability of using a reference image to suppress the spatial and temporal fluctuation. Fig. 13 is the setup schematic. The image is split into two nearly identical images by a Wollaston prism. The detection configuration is the "crossed and balanced" setup, as shown in Fig. 3; therefore, one beam is the signal beam and another beam is the reference. The polarizer after the Wollaston prism is used to attenuate the reference beam. The results are shown in Fig. 14. Fig. 14(a) shows two nearly identical images $I_{L}^{t_{1}}, I_{R}^{t_{1}}$ split from one object obtained at time $t_{1}$. Fig. 14(b) is the difference image $I_{L}^{t_{2}}-I_{L}^{t_{1}}$ of two successive images. Ideally, if there was no fluctuation, Fig. 14(b) would be all zeros. However, there do exist temporal and spatial fluctuations. The spatial fluctuation is not completely random, but there exists spatial coherence. 

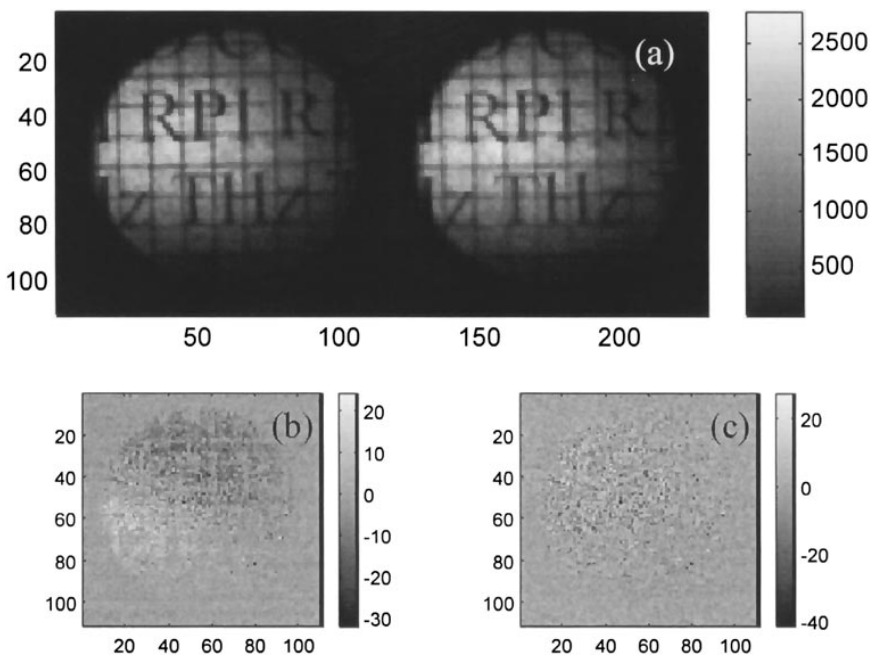

Fig. 14. Suppression of the laser fluctuation by real-time reference. (a) Two identical images are split by a Wollaston prism. (b) Noise picture without and (c) with correction using real-time reference.

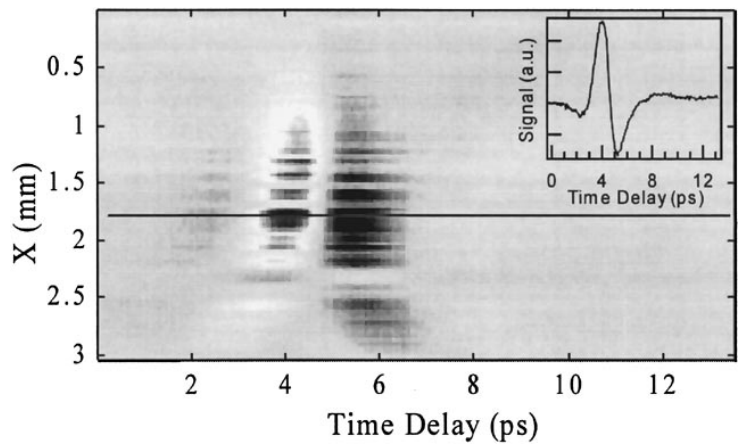

Fig. 15. Spatio-temporal terahertz imaging of an optical rectification emitter taken with a CCD operating in the "lock-in" mode. The horizontal lines come from the inhomogeneity of the EO crystal. The inset is the waveform along the solid horizontal line.

The spatial coherence is the basis for the correction because although the two split images look quite similar, it is not possible to get two identical images. Nevertheless, one image can be a good reference of the other, thanks to the spatial coherence. Mathematically, (6) is used to correct the fluctuation

$$
I_{L}^{t_{2}}-I_{R}^{t_{2}} \frac{I_{L}^{t_{1}}}{I_{R}^{t_{1}}}
$$

In Fig. 14, the corrected picture [Fig. 14(c)] is indeed more uniform than the uncorrected picture [Fig. 14(b)], indicating that the "crossed and balanced" geometry can also be used in the imaging system.

2) Software Lock-in CCD: The CCD can be operated in the "lock-in" fashion, i.e., it is synchronized to the chopper. The signal image is obtained by subtracting the image without terahertz modulation from the image with terahertz modulation. The SNR is improved by averaging. Fig. 15 plots the spatiotemporal imaging of an optical rectification emitter obtained in the dynamic subtraction with a CCD, which has a $26-\mathrm{Hz}$ frame rate and average over 200 cycles. The SNR is greatly enhanced. Due to the $1 / f$ property, it is highly desired to use

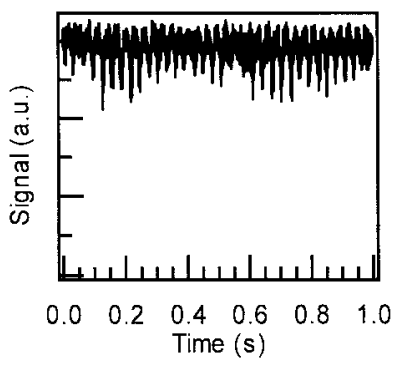

(a)

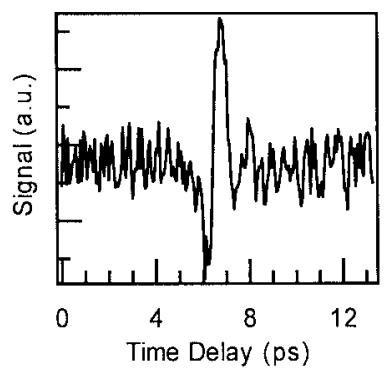

(c)

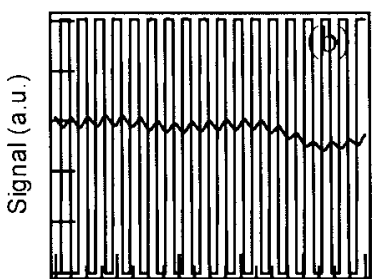

.500 .502 .504 .506 .508 .510 Time (s)

(b)

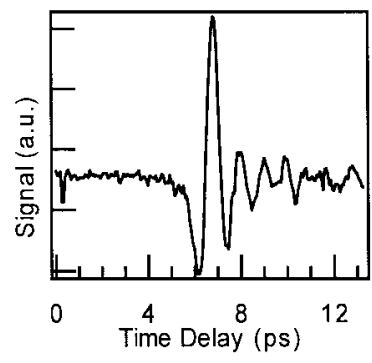

(d)
Fig. 16. Simulation of the software CCD by one single detector. (a) Photodiode output of the probe beam; the terahertz modulation is buried in large laser noise in long time scale. (b) Expanded time scale. The square wave is the chopper synchronization signal; the small sine-like curve is for the probe beam. Terahertz modulation is clearly seen, but there exists large drift. (c) Terahertz waveform measured with this simulated software lock-in with the chopper operating at $63 \mathrm{~Hz}$. (d) The same as in (c), but with the chopper operating at $1.8 \mathrm{kHz}$. In (a) and (b), the pump/probe timing is located at the main peak of the terahertz waveform.

a high frame-rate CCD. However, a CCD with $2-\mathrm{kHz}$ frame rate and greater than 12-b dynamic range is not available.

To check the frequency dependence of the noise, a single detector is used to simulate the work of the CCD. The pump beam is mechanically chopped, therefore, the terahertz beam is modulated. One photodiode is used to measure the probe beam that is modulated by the terahertz signal at the chopper frequency. Both the chopper synchronization signal and photodiode output are digitized by an A/D card and sent to the computer. The phase detection is done in the computer. Fig. 16 plots the simulation. Fig. 16(a) shows the photodiode output of the probe beam; the terahertz modulation is buried in large laser noise in long time scale. With the expanded time scale, the terahertz modulated probe beam is seen in the sine function-like curve of Fig. 16(b). The drift of the probe beam is also seen. The signal of the chopper driver is used to synchronize the computer simultaneously [square wave in Fig. 16(b)]. The terahertz signal can be extracted by proper correlation with the chopper signal. Fig. 16(c) and (d) shows terahertz waveforms measured with this simulated software lock-in detection with the chopper operating at $63 \mathrm{~Hz}$ and $1.8 \mathrm{kHz}$, respectively. The noise level at $1.8 \mathrm{kHz}$ is much smaller than that at $63 \mathrm{~Hz}$.

\section{CONCLUSION}

We have described three EO-based terahertz imaging systems. Each system has its own advantages and disadvantages. 
A rather complete discussion has been given concerning the main issues. We should note that while the scanning system is a mature technique, the CCD system is still at its early stage. The main problem with the CCD systems is the poor SNR. The SNR can be increased by either increasing the source intensity or decreasing the noise level. The increase of the source intensity is limited. A more powerful laser may help, but that will inevitably increase the cost and size of the system. Major efforts should be made on decreasing the noise.

\section{ACKNOWLEDGMENT}

The authors are grateful for the assistance from and discussions with Q. Chen and F. Sun.

\section{REFERENCES}

[1] B. B. Hu and M. C. Nuss, "Imaging with terahertz waves," Opt. Lett., vol. 20, pp. 1716-1718, 1995.

[2] D. M. Mittleman, R. H. Jacobsen, and M. C. Nuss, "T-ray imaging," IEEE J. Select. Topics Quantum Electron., vol. 2, pp. 679-692, 1996.

[3] Q. Wu and X.-C. Zhang, "Free-space electro-optic sampling of terahertz beam," Appl. Phys. Lett., vol. 67, pp. 3523-3525, 1995.

[4] _ , "Ultra-fast electro-optic field sensors," Appl. Phys. Lett., vol. 68, pp. 1604-1606, 1996.

[5] _ "Broadband detection capability of electro-optic field probes," Appl. Phys. Lett., vol. 68, pp. 2924-2926, 1996.

[6] _ "Dynamic range of an electro-optic field sensor and its imaging applications," Appl. Phys. Lett., vol. 68, pp. 3224-3226, 1996.

[7] "Design and characterization of traveling-wave electro-optic THz sensors," IEEE J. Select. Topics Quantum Electron., vol. 70, pp. $1784-1786,1997$.

[8] _ " "7 THz ultrabroadband gaP electro-optic sensors," Appl. Phys. Lett., vol. 70, pp. 1784-1786, 1997.

[9] _ "Free-space electro-optic sampling of mid-infrared pulses," Appl. Phys. Lett., vol. 71, pp. 1285-1286, 1997.

[10] A. Nahata, D. H. Auston, T. F. Heinz, and C. J. Wu, "Coherent detection of freely propagating terahertz radiation by electro-optic sampling," Appl. Phys. Lett., vol. 68, pp. 150-152, 1996.

[11] P. U. Jepsen, C. Winnewisser, M. Schall, V. Schyja, S. R. Keiding, and $\mathrm{H}$. Helm, "Detection of THz pulses by phase retardation in lithium tantalate," Phys. Rev. E, vol. 53, pp. R3052-R3054, 1996.

[12] Q. Chen, Z. Jiang, and X.-C. Zhang, "All-optical THz imaging," in Terahertz Spectroscopy Applicat., SPIE's Photon. West'99, vol. 3617-14, San Jose, CA.

[13] Q. Wu, T. D. Hewitt, and X.-C. Zhang, "Electro-optic imaging of terahertz beams," Appl. Phys. Lett., vol. 69, pp. 1026-1028, 1996.
[14] Z. G. Lu, P. Campbell, and X.-C. Zhang, "Free-space electro-optic sampling with a high-repetition-rate regenerative amplified laser," Appl. Phys. Lett., vol. 71, pp. 593-595, 1997.

[15] _ "Coherent detection of intense freely-propagating terahertz radiation and its imaging applications," J. Chinese Inst. Elect. Eng., vol. 44, no. 33, pp. 227-238, 1997.

[16] Z. Jiang and X.-C. Zhang, "Electro-optic measurement of THz pulses with a chirped optical beam," Appl. Phys. Lett., vol. 72, pp. 1945-1947, 1998.

[17] _ "Single-spot spatial-temporal THz field imaging," Opt. Lett., vol. 23 , pp. 1114-1116, 1998

[18] "Chirped pulse measurement techniques of spatio-temporal distribution," IEEE Trans. Microwave Theory Tech., to be published.

[19] M. Born and E. Wolf, Principles of Optics, 6th ed.. Cambridge, U.K. Cambridge Univ. Press, 1980, ch. 8.

[20] Z. Jiang, F. G. Sun, Q. Chen, and X.-C. Zhang, "Electro-optic sampling near zero optical bias point," Appl. Phys. Lett., vol. 74, pp. 1191-1193, 1999.

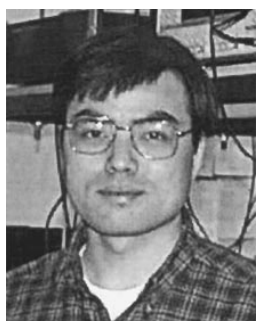

Zhiping Jiang received the M.Sc. and Ph.D. degrees in physics from the National University of Defense Technology (NUDT), Changsha, China, in 1986 and 1996, respectively.

In 1996, he was a Visiting Scholar at the Electrotechnical University, St. Petersburg, Russia. From 1996 to 1997 , he was a Post-Doctoral Researcher at the Institute of Solid State Research, Juelich, Germany. In 1997, he joined the Rensselaer Polytechnic Institute, Troy, NY, as a Research Associate. He is currently the Principal Investigator of the ZOmega Technology Corporation, Latham, NY, and a Research Assistant Professor at Rensselaer Polytechnic Institute. His current involvement is mainly with terahertz technology.

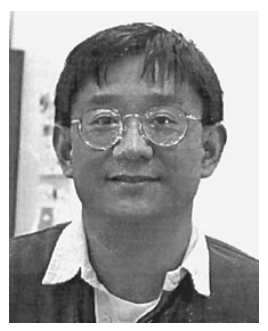

Xi-Cheng Zhang (M'91-SM'97) received the M.Sc. and Ph.D. degrees in physics from Brown University, Providence, RI, in 1983 and 1986, respectively.

In 1985, he was a Visiting Scientist at the Massachusetts of Technology, Cambridge, MA From 1985 to 1987, he was with the Physical Technology Division, Amoco Research Center From 1987 to 1991, he was with the Electrical Engineering Department, Columbia University. Since 1992, he has been with the Physics Department, Rensselaer Polytechnic Institute, Troy, NY. He is currently a Professor of physics and a Professor of electrical engineering. His current involvement is mainly with terahertz technology. 